\title{
Analytische Psychologie
}

Helmuth Remschmidt

\section{Autismus: Erscheinungsformen, Ursachen, Hilfen}

\author{
Verlag C.H. Beck, München 2000 \\ 112 S., DEM 14,80/ATS 108,-/EUR 7,50 \\ ISBN 3-406-44747-3
}

Helmuth Remschmidt, der führende Vertreter der universitären Kinder- und Jugendpsychiatrie in Deutschland, stellt in einem handlichen Taschenbuch die verschiedenen autistischen Syndrome dar, deren Epidemiologie und Hypothesen über ihre Ursachen, und er geht auf verschiedene, vor allem verhaltenstherapeutisch orientierte Behandlungsmöglichkeiten ein. In einem Anhang werden die sozialrechtliche Zuordnung des autistischen Syndroms erläutert und eine Auswahl von allgemeinverständlichen Büchern und wissenschaftlicher Spezialliteratur zum Autismus angegeben.

Mir ist unklar geblieben, an welche Leserschaft sich die Schrift richtet. Sie ist in der Art eines wissenschaftlich abgespeckten Handbuchartikels auf der Grundlage einer umfassenden Datenbankrecherche der wissenschaftlichen Literatur zum Autismus geschrieben. Sie richtet sich also vermutlich nicht an Laien oder betroffene Familien, dazu ist sie zu fachspezifisch geschrieben. Für Kinder- und Jugendpsychiater oder Kinder- und Jugendlichenpsychotherapeuten ist das Buch geeignet, wenn man sich kurz, aber präzise über neuere Forschungsergebnisse vor allem zur Genetik, Neuropsychologie und Biochemie informieren will - nicht aber, wenn man vertiefendes Wissen zur Psychotherapie (unabhängig von der Art des Verfahrens) dieser nach wie vor rätselhaften Störung erwartet.

In dem Kapitel «Was hat sich bewährt in der Behandlung autistischer Störungen?» werden zwar über 25 Seiten die Ergebnisse einer Analyse von «727 Literaturstellen zur Therapie» seit 1996 übersichtlich zusammengefasst und auch angemessen bewertet, doch darf man nicht erwarten, Informationen zu erhalten, die über sehr allgemeine Feststellungen hinausgehen, die ebenso für eine Reihe anderer Störungen gelten können.

Was ich an dem Buch vermisse, ist, dass eigentlich an keiner Stelle etwas von dem spezifischen Erleben und der Beziehungsstörung in der Begegnung mit autistischen Menschen spürbar wird. So finde ich es auch bedauerlich, dass der Autor ein altes Denkmuster wiederholen muss, das leider unter Kinder- und Jugendpsychiatern weit verbreitet ist, wenn er schreibt: «Mit zunehmendem Wissen über die biologischen Ursachen des Autismus verloren psychodynamische Theorien und die daraus abgeleiteten Therapieverfahren an Bedeutung» (p. 69). Ein Ergebnis der im Buch dargestellten (nichtpsychodynamischen) Forschung ist ja gerade, dass es eben noch keine befriedigende Ursachenerklärung gibt, auch keine biologische. Zum anderen wird ignoriert, dass sich auch psychody- 
namische Therapien bei schweren Störungen in den letzten 20 Jahren behandlungstechnisch grundlegend verändert haben - was nicht unbedingt damit gleichzusetzen ist, dass psychodynamische Theorien die Ursachen des Autismus erklären können! Um sich darüber zu informieren, hätte z.B. ein Blick in das in London erscheinende «Journal of Child Psychotherapy» oder in Publikationen des Tavistock-Instituts genügt. Die moderne psychoanalytische Kinder- und Jugendlichenpsychotherapie bezieht Forschungen aus anderen Grundlagenbereichen in ihr Ursachenverständnis mit ein und hat sich weitgehend von einem ursachenorientierten zu einem beziehungsanalytischen Denken entwickelt; dies aber ist im therapeutischen Umgang mit oder in der Beratung von autistischen Kindern, Jugendlichen und deren Familien von grundlegender Bedeutung.

Mit dieser Einschränkung möchte ich das Buch wegen seines sachlichen und auf angenehme Weise aufklärerischen Impetus jenen Psychotherapeuten, Sozialpädagogen und Kinderärzten empfehlen, die sich über das Störungsbild informieren wollen, wenn sie Beratungskontakte zu Familien mit autistischen Angehörigen haben.

Gustav Bovensiepen, Köln

Theodor und Angela Seifert

\section{So ein Zufall! Synchronizität und der Sinn von Zufällen}

Herder, Freiburg 2001

220 S., DEM 38,-

ISBN 3-451-27552-X

Es ist Mittwoch, der 15. 5. 08.05 morgens. Eben hat ein Patient angerufen und gesagt, er stünde im Stau und würde 20 Minuten später kommen. An den Vortagen habe ich darauf geachtet, ob sich irgendwelche Dinge ereignen, die sich als Synchronizitäten interpretieren liessen und die ich bei der Rezension des Buches von Angela und Theodor Seifert berücksichtigen könnte. Es war mir bislang aber nichts Diesbezügliches aufgefallen. Jetzt fällt mir ein, es könnte doch ganz spannend sein, das Weisheits- und Orakelbuch I Ging zu befragen, das ja auf einer synchronistischen Weltsicht beruht. Ich hole meine chinesischen Münzen, stelle mich auf die Frage ein: «Was meint das I Ging zum Thema Synchronizität?» und werfe. Ich wundere mich, dass ich fast nur Achter bekomme und kein Wandlungszeichen. Schliesslich ergibt sich das Hexagramm Nr. 15, Die Bescheidenheit, ohne Wandlungszeichen. Im Urteil heisst es u.a.: «Die Schicksale folgen festen Gesetzen, die sich mit Notwendigkeit auswirken. Aber der Mensch hat es in der Hand, sein Schicksal zu gestalten, je nachdem er sich durch sein Benehmen dem Einfluss der segnenden oder zerstörerischen Kräfte aussetzt. Wenn der Mensch hoch steht und sich bescheiden zeigt, so leuchtet er im Licht der Weisheit. Wenn er niedrig ist und sich bescheiden zeigt, so kann er nicht übergangen werden. So gelingt es dem Edlen, sein Werk zu Ende zu führen, ohne sich des Fertigen zu rühmen». Eingebettet ist das Zeichen in die Zeichen Nr. 14, Der Besitz von Grossem und Nr. 16, Die Begeisterung. Dort heisst es: «Wenn man Grosses besitzt und bescheiden ist, so kommt sicher Begeisterung». Jetzt erst fällt mir das heutige Datum und die Uhrzeit auf: die 15 und die 5 wiederholen sich 
(die Zahl 5 ist zur Zeit meine Lieblingszahl, sie ist für mich vor allem die Zahl der Quintessenz, des ganzen Menschen). Was soll ich nun davon halten? Darf ich mir aus all dem einen Sinn konstruieren, der mir die Bedeutung der Synchronizität erhellt oder war alles nur reiner Zufall? Was auch immer es sein mag: Das I Ging vermittelt mir für die Rezension einige zentrale Stichpunkte zum Thema, denen ich gerne folgen möchte.

Das zufällige Finden einer inspirierenden Textstelle in einem Buch, der ungeplante, aber zur gleichen Zeit stattfindende Telefonanruf ( Das muss Gedankenübertragung sein!»), das überraschende Begegnen eines Bekannten an unwahrscheinlichem Ort, «als man gerade an ihn dachte», die ähnlichen Gefühle, Phantasien, Traummotive in Phasen einer intensiven therapeutischen Beziehung, das ahnende Vorausträumen kritischer Ereignisse - alles dies sind Phänomene, die C.G. Jung mit dem das Kausalitätsprinzip ergänzenden Prinzip der Synchronizität, dem ursachelosen, durch einen gemeinsamen Sinn Angeordnetsein von Ereignissen, zu erfassen suchte.

Dieses Konzept wird von Kritikern oft als sehr problematisch angesehen, weil es einer abergläubischen und fatalistischen Einstellung Vorschub leisten könnte, und auch Analytische Psychologen schenkten ihm bislang nur wenig positive Aufmerksamkeit, weil es dabei scheinbar um relativ seltene Phänomene geht, mit denen man im Alltag nicht allzu viel anfangen kann. Beiden Einstellungen begegnen die Autoren mit einer Fülle von erstaunlichen Erlebnissen, Erfahrungen und Einsichten. Den ersten begegnen sie mit einer bescheidenen, vorsichtigen Einstellung diesen Phänomenen gegenüber, den zweiten mit einer anspornenden Begeisterung, indem sie zeigen, dass synchronistische Ereignisse viel häufiger und variantenreicher wahrgenommen werden können, als man denkt, und dass ihr Einbeziehen in das alltägliche Leben sehr hilfreich, inspirierend und bereichernd sein kann.

Bescheidenheit: Sein Schicksal schöpferisch und günstig zu gestalten, indem wir achtsam werden für die segnenden (siehe Hexagramm 15 des I Ging), d.h. sinn- und orientierungsstiftenden Kräfte der Synchronizität; das scheint auch das zentrale Anliegen des Buches von Angela und Theodor Seifert zu sein. Es ist kein primär wissenschaftliches oder theoretisches Werk - auch wenn natürlich an verschiedenen Stellen auf Versuche einer wissenschaftlichen Annäherung an diese Sichtweise eingegangen wird. Es soll vor allem ein lebenspraktisches Buch sein, das den Lesern Mut machen will, mit Hilfe einer höheren Sensibilität für synchronistische Ereignisse eine neue Sicht der Welt und des persönlichen Erlebens kennen zu lernen und Schritt für Schritt einzuüben. Das gelingt ihnen, wie ich finde, in hohem Masse.

Synchronistische Erfahrungen können uns ermutigen, warnen, inspirieren, öffnen, lassen uns tiefere Lebensdimensionen erahnen, sie schenken uns schöpferischen Spielund Freiraum für bislang unvermutete Zusammenhänge. Dies aber nur, wenn wir «bescheiden» sind, wenn wir bereit sind, uns selbst mit unseren festgefahrenen Vorstellungen ein wenig zurück zu nehmen und auf die vielfältigen symbolischen Hinweise und Reaktionen, die uns gleichzeitig von innen und aussen entgegentreten, zu achten. Offenheit, Achtsamkeit, Aufmerksamkeit sind von daher auch die «Hauptmethoden», mit denen wir uns die zunächst vielleicht noch wundersame Dimension der Synchronizitäten erschliessen können. Das erinnert natürlich nicht zufällig stark an das taoistische «WuWei», das spontane (Nicht-)Handeln in stimmiger Ubereinstimmung mit dem Tao.

Besitz von Grossem: Die Bescheidenheit wird vom I Ging in einer Beziehung zum «Besitz von Grossem» gesehen. In einer Sensibilität und Offenheit für synchronistische 
Ereignisse zu leben, vermittelt das Gefühl der Einheit und Verbundenheit mit dem «Grossen Ganzen» des Lebens und der Schöpfung. In Jungs Definition der Synchronizität als «Schöpfungsakte in der Zeit» wird ja vor allem auch das Schöpferische der Synchronizität betont, das Schöpferische, das aus den Fesseln der alltäglichen Konvention, der ausschliesslichen Linearität der Zeit und der Kausalität erlöst und einen Raum der Freiheit eröffnet. Im Auf- und Annehmen der synchronistischen Erfahrung verbinde ich mich mit dem Schöpferischen des Lebensstroms, der gerade zu diesem Zeitpunkt sinnstiftend in das persönliche Leben eintritt.

Synchronistische Ereignisse werden von den Autoren sogar auch als «kleine Erleuchtungen» bezeichnet, weil sie neben völlig unerwarteten neuen Perspektiven dieses Gefühl von Einheit mit sich bringen können und meist auch einen numinosen Charakter haben. Oft bilden synchronistische Erfahrungen Neuorientierungen und Wendepunkte des Lebens. Man ist häufig von ihnen stark betroffen und stellt sich automatisch die Frage nach dem besonderen Sinn. Dieser Sinn ist aber kein objektiver, allgemeingültiger Sinn, sondern es bedarf der persönlichen Kreativität des Einzelnen, sich seinen stimmigen Sinn «zu konstruieren» und seiner Bereitschaft, sich «als Architekt seines eigenen Lebens» zu verstehen. Hier liegt das Freiheitsmoment, natürlich auch die Möglichkeit wie bei allen Wahlen - des Irrtums und der Selbsttäuschung. Damit wird aber auch das Subjekt und das meist verachtete oder nur verschämt erwähnte Subjektive in den Mittelpunkt gerückt und in seiner Würde endlich wieder hergestellt. Das Individuelle, Persönliche und Einmalige verbindet sich hier mit dem Unpersönlichen und Archetypischen, stets verbunden mit der Zeit als zentralem Bezugspunkt, z.B. auch der Kairos bis hin zum «ewigen Nun» der Mystiker.

Darüber hinaus bezeichnen die Autoren die Erleuchtungs- und Unus-MundusErfahrungen der Mystiker auch als «Grosse Synchronizität», weil sich in ihnen die Gegensätze des Lebens, von Zeit und Raum, Geist und Materie ebenso aufheben, wie in den kleinen «alltäglichen» Synchronizitäten.

Begeisterung: Das ist ein Wort, das ich im Gespräch mit Angela und Theodor Seifert über die Synchronizität mehrfach gehört habe, und diese Begeisterung, etwas Wertvolles gefunden zu haben und vermitteln zu wollen, ist auch in ihrem ansprechend gestalteten und anregend geschriebenen Buch auf jeder Seite spürbar. Die Perspektiven, die sich durch ein Ernstnehmen der Synchronizität eröffnen, können ja auch wirklich begeistern, selbst dann, wenn man eher kritisch und skeptisch bleiben will und man sich das Prinzip der Kausalität - gegen die im Buch nirgends polemisiert wird - als ausschliessliches Denkschema bewahren möchte. Denn es bleibt ja immer noch der spielerischkreative Umgang mit der Synchronizität, das «Tun als ob». Man könnte ja einfach mal so tun, «als ob» es doch kein reiner Zufall wäre und herauszufinden suchen, was sich unter dieser Sichtweise an Neuem ergeben könnte. Man muss nicht unbedingt an sie glauben und kann doch von ihr profitieren, ähnlich, wie wir auch von der Auseinandersetzung mit anderen symbolischen Ereignissen, die ja häufig auch nur potentielle Möglichkeiten darstellen, profitieren.

Ich bin überzeugt, dass jeder, der dieses Buch durcharbeitet, grosse Lust verspürt, ab sofort wieder stärker auf synchronistische Ereignisse zu achten und sich den sich damit entwickelnden Sinn für die schöpferische Verbindung mit dem Ganzen des Lebens nicht mehr nehmen lassen will.

Lutz, Müller, Stuttgart

Buchbesprechungen 
Ernst Spengler

\section{Psychotherapie und das Bild vom Menschen}

Ontologie, Erkenntnistheorie und wissenschaftliche «Objektivität»

Daimon, Einsiedeln 2000

280 pp., broschiert, CHF 25.-/DEM 28.-

ISBN 3-85630-604-8

Ernst Spenglers Anliegen ist es, das «Eigentliche des Menschseins» (p. 10) in Abgrenzung von «anderem Sein, nämlich sachhaftem, aber auch organischem, und ganz besonders von animalischem Sein» zu bestimmen. Dies soll die Beantwortung der Frage «Wie ist der Mensch?» - und damit die Bestimmung eines Menschenbildes - ermöglichen, das als Grundlage angemessenen therapeutischen Handelns angesehen wird. Er stützt sich hierfür auf die Arbeiten von Wilhelm Keller, in denen das tradierte «SelbstObjekt-Denken» verworfen wird. Diesem wird eine «neue Seinslogik» (p. 15) gegenübergestellt: «Kellers Methode besteht im Zurückfragen ins Grundsätzliche und dieses spricht er zunächst als These aus: Die spezifische Seinsweise des Menschen im Unterschied zu allem anderen Seienden, (...), ist Selbstsein.»

Der Autor führt weiter aus: «(...) ich kann noch so sehr versuchen, es als ein Apriorisches zu denken, also als ein Sein an sich, und es damit als etwas von mir Unabhängiges voraussetzen - es hilft nichts, denn auch dieses vermeintliche Sein an sich ist doch unvermeidlicherweise von mir gedacht und gemeint und setzt somit mein Sein schon voraus.» Daraus folgt für Spengler, dass menschliches Sein notwendigerweise stets ein Verhältnis zu sich selber einschliesst. «Diese Dialektik, diese zirkelhafte Reziprozität ist der Schlüssel zum adäquaten Seinsverständnis.» Erkenntnis ist dementsprechend nur im Rahmen einer «unumgänglichen Subjektivität» möglich - ausserhalb dieses Rahmens sei an eine Erkenntnismöglichkeit über das Psychische nicht zu denken. Das bedeutet für den Autor, eine Psychotherapie, die den Phänomenen «menschlichen Seins» Begriffe aus dem Bereich des «sachlich-dinglichen» oder «animalisch Seienden» überstülpe, durch eine «Seinstherapie» - die auf eine «Seinsdynamik» (statt Psychodynamik) zurückgreifen könne - zu ersetzen. Diesen besonderen Blickwinkel entdeckt er in der Theorie C.G. Jungs.

In nun folgenden Ausführungen setzt sich der Autor - exemplarisch dargestellt an der Psychotherapieforschung - mit der positivistischen, am Forschungsideal der Physik orientierten, Wissenschaftstradition auseinander. Hier beschreibt er die problematischen Zusammenhänge zwischen einer unzureichenden Methodik der Erkenntnisgewinnung in der Forschung und dem tendenziösen Umgang mit Ergebnissen durch Kostenträger und politische Gremien. Er schildert, wie «Wissenschaftlichkeit» dazu missbraucht wird, um macht- und standespolitischem Begehren zum Durchbruch zu verhelfen (p. 41). Dabei weist Spengler darauf hin, dass die naturwissenschaftlichen Forschungsideale in der Physik in neueren Paradigmen als (teilweise) überholt anzusehen seien. Er fordert - basierend auf der Erkenntnis, dass die Subjektivität des Forschers nicht ausgeschlossen werden könne -, dass das Handlungswissen durch die Psychotherapieforschung optimiert werden solle. Durch diese andere Methodik eröffne sich auch die Möglichkeit, über die dabei wirksamen Menschenbilder und nicht nur über «theoriefreie Deskriptionen» zu sprechen. 
Daran anschliessend erwähnt Spengler kritisch jene wissenschaftstheoretischen Positionen, denen er vorwirft, im Rahmen naiv positivistischer Annahmen die Hoffnung auf eine objektive Erkenntnis über einen Forschungsgegenstand zu hegen. Er weist in diesem Zusammenhang auf die konstruktivistischen Funktionen des Gehirnes bei Wahrnehmung und Denken hin, wodurch er die Hoffnung auf eine objektive Erkenntnis auch in der menschlichen Wahrnehmungsbegrenzung für überholt erklärt. Notwendig werde gerade die Konzeptualisierung des a priori anzunehmenden subjektiven Faktors, und er stellt dafür das Denken W. Kellers näher vor, das in der Tradition Heideggers und Husserls steht.

Die Bedeutsamkeit von Kellers Theorie sieht Spengler in dessen spezifischer Begriffsverwendung, die z.B. «Trieb» und «Libido» für das Verständnis eines dynamischen und spezifisch menschlichen Seins nicht anbieten könnten. Es sei dagegen wichtig zu zeigen, dass sich alle inhaltlich bestimmten, konkreten Lebensphänomene des «Daseins» auf die optimale Erfüllung seiner Möglichkeiten ausrichten würden. «Dies bekundet, dass der Mensch wesensmässig auf ein Sollen hin, nämlich auf die Ausgestaltung seines individuellen Seins, in Pflicht genommen ist» (p. 127). Diese nun weiter ausgeführten Konzepte werden vorgestellt, um ihre Erklärungsvielfalt gerade im Zusammenhang mit neurotischen und psychosomatischen Erkrankungen - als Beispiele gestörter Selbstverwirklichung - nachzuweisen.

Der Autor widmet sich einem hochkomplexen und hochdifferenziert diskutierten Themenbereich. Die Auseinandersetzung mit den impliziten Menschenbildern in der Psychotherapie ist eine wichtige Aufgabe. Die handlungsleitende Bedeutsamkeit wird gerade in den Vorstellungen von «krank» und «gesund», «normal» und «verrückt» der Therapierichtungen deutlich und bildet die Grundlage jedes Forschungsparadigmas. Spengler geht in seinem Buch leider nur sehr wenig auf die grosse Bandbreite wissenschaftstheoretischer Positionen und deren Menschenbilder ein.

Es wurde aber spätestens im sogenannten Positivismusstreit durch die Vertreter der «Frankfurter Schule» nachgewiesen, dass jeder Theoriebildung immer schon ein Erkenntnisinteresse vorausgehe und dass Theorien von Herrschaftsinteressen durchsetzt seien. Es wurde von dieser Gruppe die Bedeutsamkeit der gesellschaftlichen Eingebundenheit des Forschers betont, was zu einem dialektischen Mitbedenken des Verhältnisses zwischen Forschung, Gesellschaft und Wissenschaft führen müsse.

Offen bleibt, warum der Autor den Ansatz W. Kellers präferiert. So bleiben auch kritische Stimmen gegenüber dieser erkenntnistheoretischen Position unerwähnt. Zu nennen ist hier Adorno: «Die zeitgenössischen Seinsphilosophien (...) hypostasierten dabei das oberste Resultat subjektiv-begrifflicher Abstraktion, Sein, und sind damit, wie ihrer Stellung zur Gesellschaft, so auch dem theoretischen Ansatz nach, im Idealismus gefangen geblieben, ohne dessen innezuwerden» [in Albert K: Einführung in die philosophische Mystik. Darmstadt, Wissenschaftliche Buchgesellschaft, 1996, p. 173]. Weitere Kritik stammt von W. Stegmüller, der in dem Begriff «sein» lediglich ein Hilfszeitwort sieht. Er schreibt aus dieser Sicht: «Nichtbeachtung dieser Vieldeutigkeit und gedankenloser substantivistischer Gebrauch dieses Hilfszeitwortes haben eine Krankheit erzeugt, die sich bereits vor über hundert Jahren in der mitteleuropäischen Philosophie auszubreiten begann und deren Kulminationspunkt möglicherweise bereits überschritten ist: die Seinspest (das Sein des Seienden usw.).» [ebd., p. 173]. 
Obwohl der Autor in guter und umfangreicher Art in das Denken dieser Tradition einführt, habe ich den Eindruck, dass ein Ausschliesslichkeitsanspruch aus dieser philosophischen Position nicht abzuleiten ist - und dieser Eindruck entsteht bei mir auf Grund der doch zu geringen kritischen Reflexion dieser Denktradition.

Der Streit um die adäquate Annäherung und Erforschung psychischer Phänomene ist sicher so alt wie die Psychologie selbst (wenn nicht schon älter), doch zum Abschluss möchte ich Karl Jaspers zitieren, der das Problem 1923 folgendermassen beschreibt: «Es gibt Forscher, die die Neigung besitzen, diese Erkenntnisquellen für die Wissenschaft zu leugnen, die nur das sinnlich Wahrnehmbare als solches, nicht das durch das Sinnliche hindurch Verstandene als objektiv gelten lassen wollen. Dagegen ist nichts einzuwenden, insofern man nicht mehr einen Beweis für die Berechtigung einer letzten Erkenntnisquelle bringen kann. Aber man kann unter allen Umständen Konsequenz fordern. Diese Forscher müssen, um widerspruchslos zu bleiben, aufhören, von Seelischem überhaupt zu reden, an Seelisches als Wissenschaftler überhaupt zu denken, sie müssen aufhören, Psychopathologie zu treiben, sich vielmehr auf Hirnprozesse und körperliche Vorgänge bei ihrem Studium beschränken. (...) Solche Konsequenz könnte Achtung erzwingen und wäre eines Forschers würdig; das hartnäckige Bestreiten und Zweifeln durch prinzipielle Einwände, wie z.B. das alles sei bloss subjektiv und dergleichen, ist unfruchtbarer Nihilismus.» [Jaspers, 1923, zit. nach Kriz J. et al.: Wissenschafts- und Erkenntnistheorie. Opladen, Leske \& Budrich, 1996, p. 135]

Ernst Spengler gibt dem Leser eine Menge Denkaufgaben auf. Er lädt durch die Gestaltung seines Buches zur reflektierenden Bearbeitung eigener Positionen ein.

Carsten Caesar, Berlin

Ernst Spengler

\section{Psychotherapie und das Bild vom Menschen}

Ontologie, Erkenntnistheorie und wissenschaftliche «Objektivität»

Daimon, Einsiedeln 2000

280 pp., broschiert, CHF 25.-/DEM 28.-

ISBN 3-85630-604-8

Es gibt verschiedene Ausprägungen der psychotherapeutischen Konzepte und Schulen. Ihnen allen liegt ein - mehr oder weniger explizit formuliertes - Menschenbild zu Grunde. Das Bild vom Menschen bestimmt aber nicht nur die jeweilige Therapierichtung, sondern auch die Menschen, die darüber ein Buch schreiben.

Spengler bezieht sich bei der Darstellung des Menschenbildes, das hinter der Psychoanalyse Jungscher Prägung steht, auf die philosophisch-anthropologische Analyse des Seins, das in einer unauflöslichen dialektischen (dialogischen?) Bezogenheit zu allem anderen Seienden steht, wie es von Wilhelm Keller vertreten und ausgearbeitet worden ist.

Damit leistet der Verfasser einen wichtigen Beitrag zu der Frage, welche Form der Wissenschaftlichkeit der Psychotherapie angemessen sei. Er weist überholte naturwissenschaftliche Paradigmen und entsprechende Forschungsdesigns für die Psychotherapie 
zurück und verweist auf eine originär ontologische, geisteswissenschaftliche Position als Grundlage von Psychotherapie, in Auseinandersetzung mit all dem, was auch noch ist in dieser Welt, also auch den verschiedenen naturwissenschaftlichen Ansätzen. Ein respektvolles Miteinander müsste entstehen. Dies ganz besonders in der Therapieforschung. Es geht Spengler keineswegs darum, den Erweis der Wissenschaftlichkeit der Psychotherapie zu verweigern, er wendet sich vielmehr dagegen, dass nur eine Form der Wissenschaft im Zusammenhang mit Psychotherapie die Wahrheit für sich beansprucht, vor allem wendet er sich dagegen, dass einige Interessenvertreter bestimmen wollen, was in diesem Zusammenhang wissenschaftlich ist, und das dann für standespolitische Zwecke missbrauchen. Der Standespolitiker Spengler spricht aus jahrelangen standespolitischen Erfahrungen.

Auf welches Menschenbild bezieht sich Spengler nun?

Das Wesentliche am Menschenbild, das auf der philosophisch-anthropologischen Analyse des Seins beruht, ist die Überzeugung, dass Selbstsein die Aufgabe des Menschen ist, dem Menschen aufgegeben.

Erkenntnistheoretisch bezieht sich Spengler darauf, dass «die Wirklichkeit ein Konstrukt des menschlichen Seins» (p. 94) ist. Er verweist darauf, dass sich darin der moderne Hirnforscher Roth, der Psychotherapeut Jung und der Seinsphilosoph Keller einig sind. Natürlich gibt es die Welt. Aber es braucht ein Bewusstsein, das mit der Welt in Interaktion treten kann, damit Welt auch wirklich «ist», damit die Dinge der Welt auch etwas bedeuten. Es ist dies ein konstruktivistischer Gesichtspunkt: Im Selbstsein werden wir Menschen selbst, wird die Welt, wird das andere. Selbstsein bedeutet, dass der Mensch sich seinen Möglichkeiten entsprechend, seinem Potential gemäss entfalten muss. Bei Jung wird dies Individuationsprinzip genannt, bei Keller Expansivität des Lebens, Streben nach einem «hochwertigen eigenen Dasein (p. 123), was bei Keller unter Selbstwertstreben, aber auch Selbstverwirklichungsstreben, abgehandelt wird. Die Grundformen der menschlichen Expansivität, des Selbstverwirklichungsstrebens (ontologisch), des Selbstwertstrebens (psychologisch), vollziehen sich nach Keller in den Bereichen des Habenwollens, des Behauptungs- und Dominanzstrebens, des Kontaktstrebens und des Betätigungsstrebens (pp. 133ff.). Das gesamte Leben - so Spengler in Anlehnung an Keller - vollzieht sich im Rahmen dieser vier Grundstrebungen. Und diese vier sind überformt und durchdrungen zugleich von einem fünften Streben, dem Selbstverwirklichungsstreben. Jeder dieser Strebungen steht im gelebten Leben auch eine Hemmung dieses Strebens gegenüber. Themen der Psychotherapie wie gestörte Selbstverwirklichung, beeinträchtigter Individuationsprozess, defiziente Seinsweise - sie betreffen alle die gleiche Daseinserfahrung der Hemmung, jeweils von einer anderen Warte aus formuliert.

Diese Grundstrebungen mit ihren Hemmungen werden beschrieben und mit praktischen Beispielen aus der Psychotherapie in Verbindung gebracht. Dadurch werden bekannte Störungsbilder auch noch in einem anderen Zusammenhang aufgezeigt als üblich. Es wird deutlich: Das Idealbild eines Menschen, der sein Leben «optimal selbsthaft zu führen und zu gestalten» (p. 196) vermag, kann kein konkretes Therapieziel sein. Dieses kann vielmehr im «lebenslangen Bemühen und im Aushalten des wesenmässig bedingten Nichterreichenkönnens» (p. 196) liegen.

Spengler versucht in diesem Herzstück des Buches psychodynamische und psychotherapeutische Überlegungen und Zusammenhänge in Verbindung mit der philosophisch- 
anthropologischen Sicht des menschlichen Seins zu bringen, als der für ihn schlüssigen theoretischen Grundlage für die «reflektierte Selbstbezogenheit» des Menschen. Ungeachtet dessen, ob der Leser oder die Leserin von dieser Sichtweise zu überzeugen ist, ist sie anregend, und von ihr könnten wichtige Impulse ausgehen. Exemplarisch ist, dass im Zusammenhang mit der Jungschen Psychotherapie eine kohärente Verbindung von Menschenbild, Ziel der Behandlung, therapeutischen Konzepten und dann im letzten Teil auch von praktischer therapeutischer Arbeit dargestellt wird - auch das eine Form der Wissenschaftlichkeit.

Das Menschenbild, wie ich zu Beginn meiner Rezension betonte, bestimmt auch das, was ein Buchautor schreibt. Was also das in diesem Buch dargestellte Menschenbild betrifft: «Dazu gehört als Grenzfall des Selbstseins, dass es darauf angelegt ist, sich optimal zu realisieren, das heisst, sich im Sinne seiner eigensten Möglichkeiten zu vollziehen.» (p. 124) Auf den Verfasser des Buches angewendet: Wir treffen in diesem Buch den Berufspolitiker, den Philosophen und den Psychotherapeuten Spengler. In jeder Rolle hat er etwas zu sagen, und teilt uns, oft auch in Nebensätzen, etwas mit, was er in jahrelanger therapeutischer und standespolitischer Arbeit erfahren hat.

Verena Kast, St. Gallen

\section{J ürg Theiler \\ Dike oder die Kunst, das Richtige zu tun Instinkt, Affekt und Vernunft ins Gleichgewicht bringen}

Walter, Düsseldorf und Zürich 2000

CHF 52.50, DEM 58.-, ATS 423.00

ISBN 3-530-40102

Neuro-analytische Psychologie - das hat nichts mit Medizin zu tun, es ist ein junger, meines Erachtens überaus verheissungsvoller, Spross der Analytischen Psychologie. Näheres dazu versuche ich mit den folgenden Zeilen zum neu erschienenen Buch von Jürg Theiler, Zürich, herauszuarbeiten.

Dike ist der Name einer griechischen Göttin. Sie symbolisiert Ordnung, Gerechtigkeit und Friede. Das Buch verspricht, dass ich lernen kann, die richtigen Entscheidungen zu treffen. Es verspricht, Ordnung, Gerechtigkeit und Friede, also Dike, in meinen Entscheidungsprozess zu bringen.

J. Theiler regt an, diese Erkenntnisse auch für die Analytische Psychologie fruchtbar zu machen. Damit dies geschehen kann, muss diese die neurobiologische Wissenschaft zur Kenntnis nehmen und deren Wissen über die Struktur des Gehirns integrieren. Das Buch liefert einen sorgfältig ausgearbeiteten Vorschlag für eine solche Integration.

In einem ersten Teil werden wir eingeführt in den neurobiologischen Konstruktionsplan des Gehirns. Dabei handelt es sich um die Beschreibung eines aktuellen, wissenschaftlichen Modells.

Kurz zusammengefasst sieht das so aus: Unser Gehirn hat sich durch die Evolutionsgeschichte hindurch in drei Schritten entwickelt: 
Das Stamm- und Kleinhirn, auch Reptilienhirn genannt, ist der evolutionsgeschichtlich älteste Hirnbereich, etwa 280 Mio. Jahre alt. Seine Hauptfunktion ist das Generieren von Kraft. Theiler nennt es das «Instinkthirn».

Dann beherbergt unser Gehirn aber auch das Säugetierhirn. Das entwicklungsgeschichtlich ältere Säugetierhirn heisst limbischer Kortex oder Emotionalhirn und ist etwa 165 Mio. Jahre alt. Seine Hauptfunktion besteht im Generieren von Wille. Es wird von Theiler mit «Affekthirn» bezeichnet.

Das rund 5 Mio. Jahre alte jüngere Säugetierhirn (Neokortex oder Grosshirn) wird von Theiler mit «Vernunfthirn» bezeichnet. Damit hat es eine besondere Bewandtnis: Das Vernunfthirn besteht aus zwei voneinander unabhängigen, unterschiedlich funktionierenden Teilsystemen: der rechten und der linken Hirnhälfte.

Die linke Hirnhälfte des Vernunfthirns nennt Theiler das «instrumentelle Vernunfthirn». Es arbeitet nach dem ökonomischen Prinzip, strebt Effizienz und Effektivität an mit den Instrumenten der Logik. Es sucht nach Ursachen und erkennt deren Wirkungen, es erkennt Ordnungen und klassiert Ereignisse ohne erkennbare Ursachen als Zufälle. Seine Hauptfunktion besteht im Generieren von Wissen und Können. Es trennt das Subjekt vom Objekt, den Betrachter vom Betrachteten.

Die rechte Hälfte des Vernunfthirns funktioniert genau umgekehrt wie seine linke Zwillingsschwester. Visuell statt verbal, synthetisch statt analytisch, holistisch statt fragmentarisch, inhaltlich korrekt statt formal abstrakt, intuitiv statt logisch, zeitunabhängig statt in zeitlicher Abfolge, rezeptiv im Gegensatz zu aktiv, analog und nicht digital, und das alles hat zur Folge: Es arbeitet schnell und diffus im Unterschied zu langsam und präzise. Seine Hauptfunktion besteht im Erkennen der persönlichen Berufung. Es verbindet die BetrachterIn mit dem Betrachteten. Theiler nennt es das «empathische Vernunfthirn».

Theiler beschreibt die Funktionsweisen dieser verschiedenen Hirnareale sehr anschaulich. Dadurch wird nachvollziehbar, dass ein gerechtes und harmonisches Zusammenspielen dieser so unterschiedlichen Hirnbereiche ein anspruchsvolles Lebensziel sein kann. Meistens gelingt das Zusammenspiel nicht, und bestimmte Hirnfunktionen - von Theiler «MitarbeiterInnen» genannt - werden unterdrückt und können nur noch mit verschlüsselten Signalen auf sich aufmerksam machen. Müdigkeit und Unwohlsein, Schmerzen, Krankheiten oder Unfälle, Depressionen und Psychosen, auch Zellveränderungen und -zerfall sind Hinweise auf unterdrückte Hirnfunktionen.

Ein erfülltes Leben zu leben würde heissen, mit allen «MitarbeiterInnen» des Gehirns in Frieden und Gerechtigkeit, d.h. in einem dynamischen Gleichgewicht, zusammenarbeiten zu können.

In einem zweiten Teil bekommen wir Hinweise auf eine Strategie, wie wir die verschiedenen Hirnfunktionen angemessen erkennen und berücksichtigen können. Wenn das gelingt, dann befinden wir uns im Bereich der göttlichen Dike, der Kunst, das (für mich in diesem Moment) absolut Richtige erkennen und tun zu können.

Hier kommen Theiler seine Erfahrungen in Management, Marketing, Kommunikation und strategischer Planung zugute. Er entwirft eine konkrete Anleitung zur «Verwirklichung des Plans - eine Strategie zur Realisierung der eigenen Berufung»:

1. kennen und erkennen Sie die «MitarbeiterInnen» Ihres Hirns;

2. bestimmen Sie die stärksten und schwächsten Mitarbeiter;

3. fördern Sie die schwächsten MitarbeiterInnen; 
4. bringen Sie Ihre Mitarbeiter miteinander ins Gespräch;

5. bewegen Sie sich in Richtung des dynamischen Gleichgewichts;

6. führen Sie, ohne zu dominieren;

7. lösen Sie die Aufgaben zuerst in Ihrem Innern;

8. hören Sie auf Ihre Träume;

9. sprechen Sie mit Ihren MitarbeiterInnen.

Die Mitarbeit aller Hirnfunktionen ist notwendig, weil wir nur so unsere individuelle Berufung erkennen, das Wissen, das Können, den Willen und die Kraft aufbringen, um das zu werden, was wir sind.

Theiler unterscheidet acht Grundtypen des Verhaltens. Jeder Grundtyp des Verhaltens kann einem - vorherrschenden - Hirnbereich zugeordnet werden.

Der Kämpfer/die Siegerin lässt sich vorwiegend vom «Instinkthirn» leiten.

Die Führerin/der Realisator wird hauptsächlich vom «Affekthirn» bestimmt.

Der Perfektionist/die Innovatorin benutzt fast ausschliesslich das «instrumentelle Vernunfthirn».

Die Sehnsüchtige/der Partner überlässt sich dem «empathischen Vernunfthirn».

\section{Dike in der Psychotherapie}

Dike, der Zustand des dynamischen Gleichgewichts, in dem die eigene Berufung gefunden und verwirklicht wird, ist eine Strategie und Psychotherapieform, die der Tiefenpsychologie und da der Analytischen Psychologie zuzuordnen ist. Mit Dike will Theiler aber die Analytische Psychologie erweitern und aktualisieren, insbesondere sollen damit die jüngeren Erkenntnisse aus der Neuro-, Molekular- und Entwicklungsbiologie berücksichtigt werden. Damit einhergehen würde eine Aktualisierung der Sprache, was der Analytischen Psychologie zum Guten gereichen könnte.

Mit Dike wird transparent gemacht, welches Menschenbild, welches Krankheitsbild und welche Methode hinter dieser Therapieform stehen.

Zum Menschenbild: Vier Hirnbereiche und ihre Funktionen leiten das Verhalten des Menschen.

Zum Krankheitsbild: Durch Abweichungen vom Gleichgewicht zwischen den vier Hirnbereichen entstehen destruktive Wirkungen, die physische und psychische Symptome hervorrufen können.

Zur Methode: Eine Beteiligung aller Hirnareale und Hirnfunktionen am Entscheidungsprozess im Sinne einer Integration von allen beteiligten «MitarbeiterInnen» wird angestrebt.

Dike ist eine Bewusstseinstherapie, niemand kommt daran vorbei, niemand kann einem diese Aufgabe abnehmen. Es gibt aber Situationen, in denen Begleitung gewünscht und sinnvoll ist. Hier ist das Arbeitsfeld für neuro-analytische PsychologInnen.

Der Schluss des Buches ist überschrieben mit: «Sie, der Weg und das Ergebnis». Darin fasst Theiler zusammen, was Dike im Leben und in der Psychotherapie beinhalten kann: prozessorientiert handeln, alle Entscheidungsträger (der Hirnregionen) gleichwertig und selbstbestimmt am Entscheidungsprozess unseres Lebens beteiligen.

Ich habe das Buch von Theiler schon vor einigen Monaten zur Rezension angefordert. Dann konnte ich mich aber einfach nicht daranmachen, es zu lesen und zu rezensieren. Dike - dieses Wort verstellte mir den Zugang. Als ich ab und zu darin blätterte, sah ich die vielen Tabellen - und schreckte wieder davor zurück. Mit dem Druck im Nacken, 
endlich vorwärts zu machen mit der Rezension, und irgendeiner widerspenstigen Hirnregion in mir drin, die sich störend dagegen stemmte, verging ein Monat nach dem andern. Aber dann begann ich doch darin zu lesen und horchte auf. Da kam mir eine faszinierende Mischung aus Naturwissenschaft-Managementlehre und Analytischer Psychologie entgegen. Aha-Erlebnisse nicht nur in bezug auf die beiden Bereiche des rechten und linken Vernunfthirns blitzten in mir auf. Schliesslich steckte ich das Buch in mein Gepäck für die 8. Basler Psychotherapietage. Es wurde mir da zu einem wichtigen Inhalt. Vor dem Abschlussvortrag entdeckte ich Dr. J. Theiler in der Menge. Das freudige Erstaunen auf beiden Seiten war gross. Die Rezension habe ich zuhause im $\mathrm{Nu}$ geschrieben.

Ich denke, für analytische PsychologInnen ist es ein «Muss», sich Kenntnisse über das Funktionieren des Gehirns zu erwerben. Jürg Theilers Buch bietet sich dazu in besonderer Weise an, weil er die Ergebnisse der Gehirnforschung anschaulich und in einer überzeugenden Gesamtschau darzulegen versteht.

Ursula Bez Bühler, Maschwanden 